\title{
L'utilizzo della tecnica ad ultrasuoni FAST eseguita dagli infermieri del Dipartimento di Emergenza: una revisione sistematica
}

\author{
Nurse-performed FAST ultrasound in the Emergency Department: a systematic review
}

Matteo Storti ${ }^{1,2}$

Lorenzo Musella ${ }^{3}$

Vito Cianci 3,4

\section{RIASSUNTO}

La metodica FAST è considerata il gold standard delle valutazione point-of-care del paziente con trauma addominale chiuso tanto da aumentarne l'uso anche da parte di professionisti non solo radiologi, ma anche del personale che opera in emergenza come l'infermiere. Lo scopo di questa revisione sistematica è quello di valutare l'efficacia dell'ecografia FAST eseguita dagli infermieri di emergenza. Sono state consultate 7 banche dati di letteratura primaria e secondaria. Inoltre sono state visionate 3 riviste nazionali attinenti al settore. La revisione è stata condotta fra marzo e agosto 2011 costruendo 9 stringhe di ricerca. Gli articoli visualizzati sono stati valutati criticamente da due autori in modo indipendente. Non sono state applicate restrizioni di lingua né di tempo di pubblicazione. Complessivamente sono stati visualizzati 4767 documenti ma solo 4 pertinenti alla revisione. In totale sono state incluse 1035 ecografie FAST svolte da personale infermieristico. I risultati mostrano che l'impiego dell'ecografia FAST eseguita dagli infermieri formati è molto efficace con una sensibilità del 84\% (95\% CI 72.1-92.2) ed una specificità del 97,37\% (95\% CI 92.55-99.10). Il tempo di esecuzione pratica aveva una media di 156 secondi (2.6 minuti), tempo mediano di 138 secondi (range=76 a 357). Solo in uno studio veniva specificato in modo dettagliato il tipo di corso formativo che dovevano frequentare gli infermieri. In conclusione l'ecografia FAST eseguita dal personale infermieristico può avere un ruolo determinante nell'ambito delle emergenze e nei processi specifici di triage in quanto vero e proprio strumento di screening.

Parole chiave: FAST, infermieristica di emergenza, lesione addominale, servizio di emergenza, ultrasonografia.

\section{ABSTRACT}

Focused Assessment with Sonography for Trauma (commonly abbreviated as FAST) is considered for patients with blunt abdominal trauma as the gold standard for accident assessment in site. This method is increasingly used even by not radiologists professionals, as well as by nurses who works in emergency settings.

This systematic review is aimed at evaluating the effectiveness of ultrasound FAST performed by nurses in emergencies department. Seven databases of primary and secondary literature as well as three national journals relevant to the field were consulted. The review was conducted between March and August 2011 developing 9 search strings. Articles have been critically reviewed by two authors independently. No restriction on language or time of publication have been used.

A total of 4767 documents were displayed, of those only 4 were considered to be reviewed. A total of 1035 FAST ultrasound performed by nurses were included.

The results show that the use of ultrasound FAST performed by trained nurses is very effective, with a sensitivity of $84 \%$ (95\% CI 72.1-92.2) and a specificity of $97.37 \%$ (95\% CI 92.55-99.10). Practice execution time was an average of 156 seconds (2.6 minutes), median time of 138 seconds (range $=76$ to 357). Just one study specified the training course that nurses were required to attend. To sum up, FAST ultrasound performed by nurses have an important role in emergencies management as well as in triage setting as valid screening tool.

Key words: abdominal injuries, emergency nursing, emergency service, FAST, ultrasonography.

\section{INTRODUZIONE}

I trauma addominale rappresenta una causa di accesso 1 frequente in Pronto Soccorso (PS) e nei dipartimenti di emergenza (DE) di tutto il mondo; mentre i pazienti con trauma addominale esclusivo o nel contesto di un

1 Centro Formazione, ULSS n. 5 "Ovest Vicentino"

2 Gruppo di Ricerca, Collegio IPASVI di Vicenza

3 UOC di Pronto Soccorso - SUEM 118, ULSS n. 5 "Ovest Vicentino"

4 International Training Center (ITC) WINFOCUS Vicenza politrauma complesso sono anche oggetto di soccorso pre-ospedaliero degli equipaggi di Emergenza sul territorio (Walcher et al., 2006). Il trauma addominale suona come un'insidia costante in termini di morbilità e mortalità di questa categoria di pazienti (Walcher et al., 2006; Melniker et al., 2006). Tra le cause determinanti di outcome sfavorevoli o gestioni non ottimali, per ritardi e inadeguate centralizzazioni, l'emoperitoneo risulta essere al primo posto (Ryan et al., 1986); segue il fattore tempo che continua a giocare un ruolo determinante secondo la logica della "golden hour" che ispira 
uno dei paradigmi irrinunciabili nel management sia di base (PTC, ITLS) che avanzato (ATLS) del trauma. Conformemente, nel corso degli ultimi decenni, si è progressivamente affermato l'impiego degli ultrasuoni (US) mirati all'identificazione di raccolte fluide all'interno del peritoneo o di una delle altre cavità sierose dell'organismo come il pericardio o la pleura (Ryan et al., 1986). La Focused Assessment Sonography for Trauma (FAST) rappresenta una sorta di best practice per medici e chirurghi d'emergenza, entrando a pieno titolo nella valutazione integrata clinico-strumentale primaria nel paziente instabile (primary survey) o secondaria nel paziente stabile (secondary survey) (Rozycki et al., 1993; Lee et al., 2007; Cianci, 2009). Negli ultimi anni la FAST ha trovato la sua progressiva affermazione fino a diventare metodica standard integrata nella valutazione point-of-care del politrauma (ovvero quella valutazione eseguita nel "punto di cura" e gestione del trauma, dalla scena dell'incidente alla sala operatoria). In chiave di metodica, la FAST si concretizza in una sequenza standardizzata di finestre che esplorano alcuni punti sensibili delle sierose (pericardio, loggia epato-renale, loggia spleno-renale, tasca rettovescicale o cavo del Douglas), dove più facilmente si evidenzia la presenza di fluido nel paziente traumatizzato. Tale metodica è nata virtualmente in Europa negli anni settanta, grazie ad alcuni chirurghi tedeschi orientati alla ricerca di un metodo alternativo al lavaggio peritoneale diagnostico (DPL); di seguito ha trovato la sua attuale codifica ed implementazione negli USA ad opera di un noto studio (Rozycki et al., 1993) nel quale hanno coniato per la prima volta l'acronimo FAST. Nelle sue versioni successive la FAST ha poi acquisito una veste sempre più completa (EFAST, FAST-CRASH, FAST-ABCDE), diventando l'architrave su cui si è venuto a edificare l'intero sistema dell'ecografia in emergenza-urgenza ed in area critica (Critical Ultrasound). Dietro questa affermazione, come una sorta di paradigma nella gestione di pazienti traumatizzati e non, si cela una sorta di rivoluzione culturale che trova alcuni dei suoi punti descritti in letteratura (Cianci, 2009) e qualificanti in:

- necessità di amplificare i dati emergenti dalla valutazione clinica tradizionale, troppe volte inaffidabile ed incompleta (visual medicine);

- bisogno di agganciare alla valutazione in emergenza secondo il modello per gradini successivi (step by step) uno strumento che rispetti la codifica procedurale $\mathrm{ABCDE}$, garantendo tempi ed efficacia superiori;

- richiesta di uniformare ed omogeneizzare il più possibile gli interventi in emergenza-urgenza, utilizzando strumenti di facile impiego;

- necessità di creare una maggiore comunicazione all'interfaccia dei vari anelli della catena di sopravvivenza, promuovendo un linguaggio universale veicolato anche attraverso gli US;

- esigenza di identificare precocemente cause potenzialmente correggibili di arresto/peri-arresto cardiaco e, in generale, nel paziente critico, anticipando i tempi della diagnostica tradizionale intervenendo già sul territorio;

- necessità di ottimizzare la movimentazione e la centralizzazione dei pazienti nel flusso direzionale, dal territorio al centro, dalla periferia verso i dipartimenti di secondo-livello.

In questa ottica di potenziamento sinergico e multidisciplinare, si sta affermando sempre più la diffusione dell'ecografia da parte del personale infermieristico in diversi ambiti, secondo una logica di interazione all'interno del team di soccorso, potenziando la condivisione/sostituzione di ruoli, per lo meno ai livelli base (Cole, 2003). Questa esigenza si impone una volta di più nei soccorsi extraospedalieri, dove risorse umane $\mathrm{e}$ tecnologiche sono in genere circoscritte ma non solo. Una visione moderna e proiettata verso il miglioramento delle performance prevede un'integrazione sempre più stretta tra i componenti dell'équipe anche nella gestione intra-ospedaliera (Cianci et al., 2008). Gli orientamenti emergenti degli ultimi anni prevedono un impiego elettivamente operativo degli US nel management infermieristico, quale ausilio ad operazioni come il reperimento di accessi venosi periferici o il controllo del cateterismo vescicale (Kane et al., 2008; Miles et al., 2011). Ma in un'epoca in cui sono più presenti impulsi diretti alla maggiore professionalizzazione dell'infermiere, cominciano a rendersi evidenti impieghi alternativi, come la FAST.

\section{OBIETTIVO E DOMANDA DI RICERCA}

Il bisogno informativo a cui si è voluto rispondere è quello di individuare i possibili benefici nell'adozione dell'ecografia FAST, svolta dal personale infermieristico del DE, già nella fase iniziale di accertamento delle problematiche del paziente affetto da trauma addominale chiuso.

La ricerca è stata finalizzata per rispondere alla seguente domanda principale:

l'adozione dell'indagine ecografica FAST tra gli infermieri del DE riduce la mortalità nei pazienti affetti da trauma addominale chiuso?

\section{MATERIALI E METODI}

Criteri di eleggibilità degli studi inclusi nella revisione

Il metodo si è basato sul principio della revisione sistematica limitata all'impiego dell'ecografia FAST adottata dall'infermiere di DE allo scopo di ridurre la 
mortalità, la sottostima del codice colore ed i tempi di intervento al paziente affetto da trauma addominale chiuso. Conseguentemente è stata attivata una ricerca sistematica negli archivi di informazione biomedica concordando i criteri di eleggibilità. Sono stati inclusi tutti gli studi condotti sui soggetti che presentavano il presente disegno di ricerca: clinical trial, meta-analysis, linee guida, studi randomizzati e controllati (RCT), revisioni sistematiche, studi di comparazione, studi multicentrici e studi di validazione. Sono stati esclusi gli studi che trattavano della metodica di ecografia FAST praticata dai professionisti medici o, dall'impiego di tale metodica nei traumi non addominali chiusi. Infine, considerando il riassunto un elemento fondamentale, intrinseco di un lavoro scientifico, sono stati esclusi tutti gli studi che non disponessero di tale elemento. Nei database elettronici non sono state considerate restrizioni di tempo di pubblicazione.

\section{Caratteristiche dei pazienti da analizzare}

Pazienti di qualsiasi età, afferenti dal territorio o da strutture extra-ospedaliere, che richiedevano assistenza immediata in quanto presentavano uno o più traumi addominali chiusi.

\section{Tipo di intervento}

La variabile di intervento consiste nell'impiego dell'ecografia FAST, da parte dell'infermiere del DE, per attivare il migliore percorso di cura al soggetto interessato, in un'ottica di intervento rapido, garantendo l'appropriatezza delle prestazioni.

\section{Tipi di outcome rilevati}

Outcome primario

Ridotta mortalità dei pazienti affetti da trauma addominale chiuso intesa come efficacia ed accuratezza dell'ecografia FAST.

Outcome secondario

Diminuzione dei tempi di intervento sanitario.

\section{Metodi di ricerca per identificare gli studi}

La presente revisione sistematica è stata condotta tra Marzo ed Agosto 2011. I criteri di eleggibilità sono stati inseriti come limiti solo nella banca dati biomedica di PubMed. Per gli altri database non sono stati applicati limiti sulle stringhe di ricerca usate. In tutti i database le stringhe di ricerca sono composte da parole chiave redatte in inglese ad eccezione del Sistema Nazionale Linee Guida dove le parole chiave sono vagliate in lingua italiana.

I database elettronici consultati sono:

- Cochrane Library;

- Joanna Briggs Institute (JBI);

- PubMed;

- Cumulative Index to Nursing and Allied Health
Literature (CINAHL);

- National Guideline Clearinghouse (NGC);

- New Zealand Guidelines Group (NZGG);

- Sistema Nazionale Linee Guida (SNLG).

\section{Ricerca in altre risorse}

$\mathrm{Al}$ fine di esplorare il quesito posto, sono state revisionate le seguenti riviste italiane:

"L'Infermiere" e "SCENARIO" dall'anno 2004 fino alla loro recente indicizzazione in Cumulative Index to Nursing and Allied Health Literature.

- "L'Infermiere" (http://www.ipasvi.it/ecm/rivistalinfermiere.htm) dal 2002 in poi

2002 visionato tutti 5 numeri;

2003 visionato tutti 5 numeri;

2004 visionato tutti 2 numeri;

2005 visionato tutti i 10 numeri;

2006 visionato tutti i 7 numeri;

2007 visionato tutti i 6 numeri;

2008 visionato tutti i 6 numeri;

2009 visionato tutti i 6 numeri;

2010 visionato tutti i 6 numeri;

2011 visionato i numeri 1,2 e 3.

- "SCENARIO" (http://www.aniarti.it/scenario/) dal 2005 in poi

2005 visionato tutti i 4 numeri;

2006 visionato tutti i 4 numeri;

2007 visionato tutti i 4 numeri;

2008 visionato tutti i 4 numeri;

2009 visionato tutti i 4 numeri;

2010 visionato tutti i 4 numeri;

2011 visionato il numero 1 e 2 .

Successivamente sono stati esplorati anche i documenti della rivista "Emergency Care Journal" (http://www.cgems.it/inside3.aspx?ch=i.riv.list.ctg\&id

Cat=1.1.2.5.8) dal 2005 ad Agosto 2011.

2005 visionato tutti i numeri disponibili;

2006 visionato tutti i 6 numeri;

2007 visionato tutti i 6 numeri;

2008 visionato tutti i 4 numeri;

2009 visionato tutti i 4 numeri;

2010 visionato tutti i 4 numeri;

2011 visionato il numero 1e 2 .

In particolare, gli articoli contenuti nelle riviste italiane "Professioni Infermieristiche" (http://profinf.net/), "Assistenza Infermieristica e Ricerca (http://www.air-online.it/)" e "International Nursing Perspectives" (http://www.seuroma.com/ nursing/apps/autos.php) non sono stati visionati in formato cartaceo perché le prime due erano già indicizzate in CINAHL e PubMed da oltre 10 anni, pertanto la loro valutazione è stata fatta soltanto tramite il database nominato poc'anzi e, della terza rivista, si potevano reperire gli articoli da CINAHL dall'anno 2001 in poi. 


\section{Stringhe di ricerca impiegate dagli autori}

Per raccogliere le informazioni necessarie sono state costruite complessivamente 9 stringhe di ricerca. Tutti i termini impiegati derivano dalle parole, o loro sinonimi, del quesito di ricerca. Le prime 5 stringhe sono state composte da termini del Medical Subjects Heading (Mesh) dell'Index Medicus mentre le ultime 4 da vocaboli non inclusi nei Mesh terms.

\section{Stringhe di ricerca utilizzando i Mesh terms}

1 (("Ultrasonography"[Mesh]) AND "injuries" [Subheading]) AND "Emergency Service, Hospital"[Mesh]

2 ((("Injuries" [Subheading]) OR "Abdominal Injuries"[Mesh]) AND "Ultrasonography"[Mesh]) AND "Emergency Service, Hospital"[Mesh]

3 "Nurses"[Mesh ] AND "Emergency care, prehospital"[Mesh] AND "Ultrasonography"[Mesh] AND "Injuries"[Mesh]

4 "Nurses"[Mesh ] OR "Emergency nursing"[Mesh] AND "Ultrasonography"[Mesh]

5 (("Emergency Nursing"[Mesh]) AND "Ultrasonography"[Mesh]) AND "Nurses"[Mesh]

In ricerca libera sono state costruite le seguenti stringhe:

1 Echography AND Nurse AND Triage

2 Ultrasound AND Trauma AND Prehospital

3 Ultrasound AND Nurse AND Trauma AND Fast

4 Triage AND Ultrasound AND Trauma AND Prehospital

\section{Modalità di raccolta della documentazione e analisi}

Gli studi individuati dalle strategie di ricerca sono stati gestiti utilizzando il programma ProCite (versione 5 per Windows $\left.{ }^{\circledR}\right)$. Il processo di selezione degli studi da includere ha seguito i seguenti step: due autori prendevano visione dei riferimenti bibliografici emersi dall'esito delle differenti stringhe di ricerca; valutavano tutti i riferimenti potenzialmente rilevanti per essere inclusi nella presente revisione sistematica e dove vi era la necessità sono stati reperiti i riassunti ed i testi integrali al fine di valutare l'eleggibilità dei documenti. Se durante il processo di revisione, erano presenti pubblicazioni con dati mancanti venivano contattati gli autori del documento reperito. A seconda del tipo di disegno venivano impiegate delle griglie standard di valutazione critica dello studio. Qualora vi fossero state divergenze nell'ammissibilità di un articolo, si risolvevano attraverso un focus o coinvolgendo il terzo revisore. I dati sono stati estratti singolarmente, quindi confrontati dagli autori del presente documento.

Successivamente è stata fatta un'analisi di gruppo dei risultati e discusse le conclusioni. Non è stato ritenuto opportuno eseguire una combinazione quantita- tiva degli elementi raccolti dai vari studi inclusi, data l'eterogeneità dei documenti e del processo di ricerca condotto.

Tutti i revisori hanno potuto rivedere il testo e, le modifiche sono state inserite nella versione finale. I dati qualitativi sono presentati in forma narrativa.

Ogni autore di questa revisione lavorava in modo indipendente e conosceva l'argomento oggetto dello studio.

Modalità di individuazione dei bias presenti negli studi

Ciascuno dei due autori di questa revisione, in maniera indipendente, esplorava numerosi item per valutare l'articolo reperito. Le stringhe di ricerca sono composte da sinonimi per un migliore recall degli articoli. Tali criteri affrontavano il documento nella sua validità interna ed esterna. La metodica di valutazione era condivisa dagli autori ed era differente dal tipo di disegno dello studio reperito.

L'analisi critica di ciascuna revisione sistematica reperita veniva vagliata con la struttura della checklist QUOROM statement (Moher et al., 1999). Gli RCT venivano valutati criticamente con l'impiego dello strumento CONSORT statement (Moher et al., 2001). Gli studi trasversali, di coorte e caso-controllo venivano sottoposti a screening con lo strumento STROBE statement (von Elm et al., 2007). Le linee guida reperite con le stringhe di ricerca sono state controllate con la griglia AGREE nella sua ultima versione (AGREE II) (Brouwers et al., 2010; Brouwers et al., 2010; Cartabellotta, 2011).

\section{RISULTATI}

Di seguito vengono rappresentati gli esiti delle varie stringhe di ricerca su ciascuna banca dati; è ribadito che in nessuna banca dati sono stati applicati i limiti tranne che in PubMed.

\begin{tabular}{|c|c|c|}
\hline Nome del database & Documenti presenti & Documenti selezionati \\
\hline PubMed & 15 & 1 \\
\hline Cochrane Library & $\begin{array}{c}10 \\
\text { (di cui 1 protocollo) }\end{array}$ & 0 \\
\hline JBI & 0 & 0 \\
\hline CINAHL & 3 & 0 \\
\hline NGC & 2 & 0 \\
\hline NZGG & 0 & 0 \\
\hline SNLG & 0 & 0 \\
\hline
\end{tabular}

Box 1 - Documenti presenti e selezionati considerando la prima stringa di ricerca con termini Mesh dell'Index Medicus

Considerando la stringa del Box 1 sono stati visualizzati 30 studi. Applicando i criteri di eleggibilità descritti in precedenza è stato selezionato un documento. 


\begin{tabular}{|c|c|c|}
\hline Nome del database & Documenti presenti & Documenti selezionati \\
\hline PubMed & 27 & 0 \\
\hline Cochrane Library & $\begin{array}{c}1516 \text { (di cui 419 proto- } \\
\text { colli e 2 commenti) }\end{array}$ & 0 \\
\hline JBI & 0 & 0 \\
\hline CINAHL & 3 & 1 \\
\hline NGC & 2 & 0 \\
\hline NZGG & 0 & 0 \\
\hline SNLG & 0 & 0 \\
\hline
\end{tabular}

Box 2 - Documenti presenti e selezionati considerando la prima stringa di ricerca con termini Mesh dell'Index Medicus

Nel Box 2 sono risultati 1548 documenti ma soltanto 1 aveva le caratteristiche di soddisfare il quesito della ricerca. Tale studio però è presente anche in un'altra banca dati pertanto già selezionato in precedenza.

Dal Box 3 emerge che solamente 1 studio è stato selezionato, previo valutazione critica, dall'applicazione della stringa impiegata.

\begin{tabular}{|c|c|c|}
\hline Nome del database & Documenti presenti & Documenti selezionati \\
\hline PubMed & 27 & 0 \\
\hline Cochrane Library & $\begin{array}{c}1516 \text { (di cui } 419 \text { proto- } \\
\text { colli e 2 commenti) }\end{array}$ & 0 \\
\hline JBI & 0 & 0 \\
\hline CINAHL & 3 & 1 \\
\hline NGC & 2 & 0 \\
\hline NZGG & 0 & 0 \\
\hline SNLG & 0 & 0 \\
\hline
\end{tabular}

Box 3 - Documenti presenti e selezionati considerando la prima stringa di ricerca con termini Mesh dell'Index Medicus

\begin{tabular}{|c|c|c|}
\hline Nome del database & Documenti presenti & Documenti selezionati \\
\hline PubMed & 42 & 2 \\
\hline Cochrane Library & $\begin{array}{c}1420 \text { (di cui } 353 \text { proto- } \\
\text { colli e } 1 \text { commento) }\end{array}$ & 0 \\
\hline JBI & 3 & 0 \\
\hline CINAHL & 0 & 0 \\
\hline NGC & 1599 & 0 \\
\hline NZGG & 14 & 0 \\
\hline SNLG & 0 & 0 \\
\hline
\end{tabular}

Box 4 - Documenti presenti e selezionati considerando la prima stringa di ricerca con termini Mesh dell'Index Medicus

\begin{tabular}{|c|c|c|}
\hline Nome del database & Documenti presenti & Documenti selezionati \\
\hline PubMed & 0 & 0 \\
\hline Cochrane Library & 5 & 0 \\
\hline JBI & 3 & 0 \\
\hline CINAHL & 0 & 0 \\
\hline NGC & 19 & 0 \\
\hline NZGG & 0 & 0 \\
\hline SNLG & 0 & 0 \\
\hline
\end{tabular}

Box 5 - Documenti presenti e selezionati considerando la prima stringa di ricerca con termini Mesh dell'Index Medicus
Rispetto ai 3078 documenti selezionati 2 studi sono stati considerati validi ma uno di essi risulta ridondante in quanto emerge nella stringa precedente (Box 4).

Rispetto ai 27 documenti selezionati nessuno è stato congruo al quesito (Box 5).

Entrambe le pubblicazioni selezionate sono state estranee al quesito posto (Box 6).

\begin{tabular}{|c|c|c|}
\hline Nome del database & Documenti presenti & Documenti selezionati \\
\hline PubMed & 2 & 0 \\
\hline Cochrane Library & 0 & 0 \\
\hline JBI & 0 & 0 \\
\hline CINAHL & 0 & 0 \\
\hline NGC & 0 & 0 \\
\hline NZGG & 0 & 0 \\
\hline SNLG & 0 & 0 \\
\hline
\end{tabular}

Box 6 - Documenti presenti e selezionati considerando la prima stringa in ricerca libera senza termini Mesh

\begin{tabular}{|c|c|c|}
\hline Nome del database & Documenti presenti & Documenti selezionati \\
\hline PubMed & 46 & 1 \\
\hline Cochrane Library & 1 & 0 \\
\hline JBI & 0 & 0 \\
\hline CINAHL & 8 & 0 \\
\hline NGC & 1 & 0 \\
\hline NZGG & 0 & 0 \\
\hline SNLG & 0 & 0 \\
\hline
\end{tabular}

Box 7 - Documenti presenti e selezionati considerando la prima stringa in ricerca libera senza termini Mesh

Su 56 studi risultati nel Box 7 soltanto 1 studio ha soddisfatto i criteri di eleggibilità.

\begin{tabular}{|c|c|c|}
\hline Nome del database & Documenti presenti & Documenti selezionati \\
\hline PubMed & 4 & 2 \\
\hline Cochrane Library & 0 & 0 \\
\hline JBI & 2 & 0 \\
\hline CINAHL & 1 & 0 \\
\hline NGC & 1 & 0 \\
\hline NZGG & 0 & 0 \\
\hline SNLG & 0 & 0 \\
\hline
\end{tabular}

Box 8 - Documenti presenti e selezionati considerando la prima stringa in ricerca libera senza termini Mesh

Collegando le parole chiavi fra loro sono risultati 8 studi di cui 2 pertinenti, ma entrambi già considerati con precedenti stringhe.

\begin{tabular}{|c|c|c|}
\hline Nome del database & Documenti presenti & Documenti selezionati \\
\hline PubMed & 13 & 0 \\
\hline Cochrane Library & 0 & 0 \\
\hline JBI & 0 & 0 \\
\hline CINAHL & 1 & 0 \\
\hline NGC & 1 & 0 \\
\hline NZGG & 0 & 0 \\
\hline SNLG & 0 & 0 \\
\hline
\end{tabular}

Box 9 - Documenti presenti e selezionati considerando la prima stringa in ricerca libera senza termini Mesh 


\begin{tabular}{|c|c|}
\hline Titolo & Validation of nurse-performed FAST ultrasound. \\
\hline Autori & Justin Bowra, Sally Forrest-Horder, Erica Caldwell, Michelle Cox, Scott K. D’Amours. \\
\hline $\begin{array}{l}\text { Rivista di pubblicazione } \\
\text { ed anno }\end{array}$ & Injury, 2010 \\
\hline Popolazione e contesto & $\begin{array}{l}\text { Lo studio è stato condotto nel trauma center di primo livello dell'ospedale principale di Sidney, Nuovo Galles del } \\
\text { Sud (Australia), con un bacino di utenza di oltre } 800000 \text { persone ed una media di accessi annuale al PS di } 65000 \\
\text { pazienti. Sono stati considerati tutti i pazienti che afferivano al PS tra Settembre } 2006 \text { e Febbraio } 2009 \text { per i quali } \\
\text { veniva attivato il trauma team. I pazienti dovevano soddisfare uno o più dei seguenti criteri di eleggibilità: dolo- } \\
\text { re all'addome o segni di trauma addominale, ipotensione o tachicardia, diminuito livello di coscienza. } 8 \text { infermie- } \\
\text { ri esperti in traumatologia ed area critica vennero selezionati a partecipare allo studio. } \\
\text { Lo scopo dello studio è stato quello di determinare l'accuratezza della FAST, eseguita dal personale infermieri- } \\
\text { stico, come pratica alternativa laddove non fosse presente un medico abilitato. }\end{array}$ \\
\hline Disegno di ricerca & Studio retrospettivo. \\
\hline Risultati & 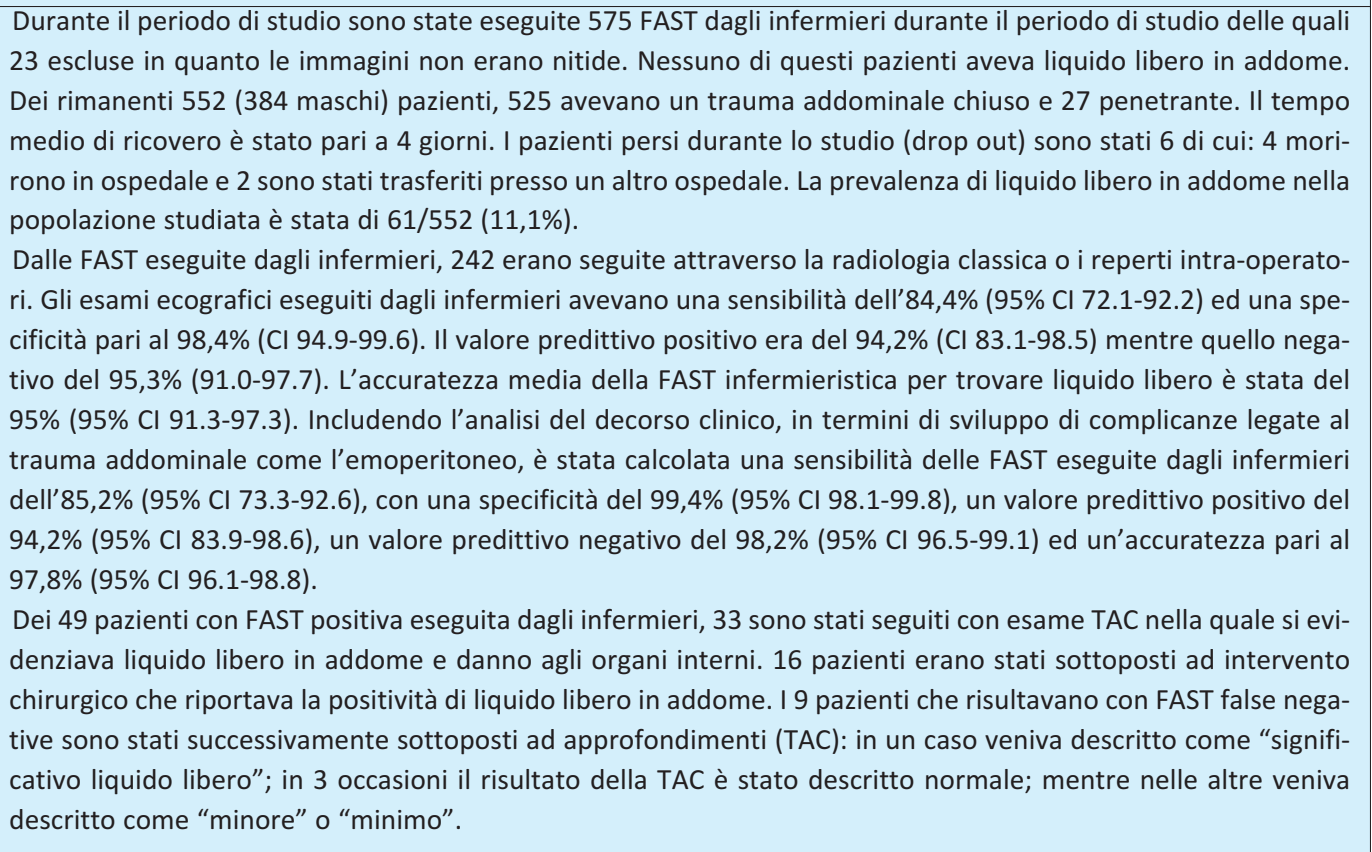 \\
\hline Discussione & $\begin{array}{l}\text { I risultati dello studio suggeriscono che l'accuratezza della FAST eseguita da infermieri esperti nella gestione del } \\
\text { trauma e, correttamente addestrati nell'eseguire tale esame, è comparabile con l'accuratezza della FAST esegui- } \\
\text { ta dai medici. } \\
\text { Studi precedentemente pubblicati dimostrarono l'affidabilità di esami ecografici eseguiti da infermieri in altri } \\
\text { campi: inserzione di cateteri venosi periferici, ecocardiografia, rilevazione di aneurismi dell'aorta addominale ed } \\
\text { ecografie ostetriche. Questo è il primo studio che analizza nello specifico gli esami FAST eseguiti da infermieri. } \\
\text { Gli autori hanno coinvolto infermieri esperti che avessero già finalità con il trauma team ed il ruolo della FAST; } \\
\text { ne consegue che gli infermieri potrebbero rendere disponibile la metodica FAST su un maggiore numero di } \\
\text { pazienti. }\end{array}$ \\
\hline Limiti & $\begin{array}{l}\text { - Dopo la dimissione il paziente non veniva monitorato pertanto potrebbe essere che alcuni di essi abbiano } \\
\text { sviluppato delle complicanze legate al trauma ed indirizzati in altri presidi ospedalieri. } \\
\text { - Il campione era di convenienza in quanto l'arruolamento si è basato sulla disponibilità e motivazione degli } \\
\text { infermieri. I risultati potrebbero essere stati diversi nel caso in cui gli infermieri coinvolti non corrisponde- } \\
\text { vano a precisi profili di esperienza e capacità tecnica } \\
\text { - Una parte dei pazienti è stata persa durante lo svolgimento della ricerca. } \\
\text { - Nella conduzione di questa esperienza non viene specificato il tipo di formazione svolta dal personale }\end{array}$ \\
\hline
\end{tabular}

Tabella 1 - Primo studio pertinente al quesito di ricerca (Bowra et al., 2010) 


\begin{tabular}{|c|c|}
\hline Titolo & Prehospital ultrasound by paramedics: results of field trial. \\
\hline Autori & William Heegaard, David Hildebrandt, David Spear, Kevin Chason, Bret Nelson, Jeffrey Ho. \\
\hline $\begin{array}{l}\text { Rivista di pubblicazio- } \\
\text { ne ed anno }\end{array}$ & Academic Emergency Medicine, 2010. \\
\hline $\begin{array}{l}\text { Popolazione e conte- } \\
\text { sto }\end{array}$ & 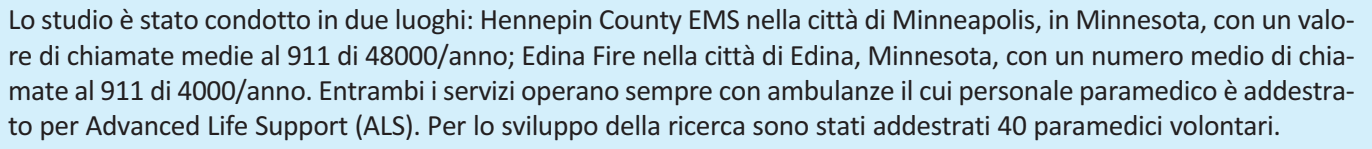 \\
\hline Disegno di ricerca & Lo studio è di tipo osservazionale prospettico. \\
\hline Risultati & $\begin{array}{l}\text { A Hennepin County sono stati addestrati } 25 \text { paramedici su un totale di } 116 \text { e soltanto } 15 \text { hanno completato l'inte- } \\
\text { ro studio. A Edina Fire, sono stati addestrati } 15 \text { professionisti su un totale di } 32 \text { e } 10 \text { hanno completato l'intera } \\
\text { ricerca. II maggiore numero di ecografie praticate sono state } 4,6 \text { con una media di } 3 \text { ecografie per professionista. } \\
\text { E' stato eseguito un totale di } 104 \text { ecografie pre-ospedaliere da Gennaio } 2008 \text { a Gennaio } 2009 \text {. I paramedici non } \\
\text { erano in grado di ottenere immagini consone nel } 7,7 \% \text { dei casi ( } 8 / 104) \text { e tutti gli esami inadeguati erano FAST. Sono } \\
\text { stati eseguiti } 20 \text { esami dell'aorta addominale interpretati come negativi dai paramedici e dal medico revisore il } \\
\text { quale era all'oscuro delle metodiche (cieco). Tutte le ecografie dell'aorta addominale erano giudicate corrette dal } \\
\text { medico revisore. } 84 \text { pazienti avevano una FAST pre-ospedaliera. Delle } 76 \text { FAST adeguate, il tempo medio per ese- } \\
\text { guirle era di } 156 \text { secondi ( } 2,6 \text { minuti). } 6 \text { (7,1\%) FAST pre-ospedaliere erano lette, da entrambi i professionisti, come } \\
\text { positive per liquido libero in addome o pericardico. In un caso un'ecografia positiva non aveva rilevanza clinica. } \\
\text { Fra gli esami ecografici pre-ospedalieri ritenuti adeguati c'è accordo nel } 100 \% \text { sull'interpretazione tra paramedici } \\
\text { e medico. Le ecografie pre-ospedaliere positive sono state confermate da reperti TAC o intra-operatori. II caso } \\
\text { falso positivo, menzionato in precedenza, è stato tenuto in osservazione e successivamente dimesso. }\end{array}$ \\
\hline Discussione & $\begin{array}{l}\text { I risultati dello studio mostrano che l'ecografia pre-ospedaliera può essere introdotta con successo in un setting } \\
\text { d'emergenza con qualità ed accuratezza anche se eseguite da personale paramedico addestrato correttamente } \\
\text { dal medico esperto in ecografia d'urgenza. Lo studio ha dimostrato che le ecografie pre-ospedaliere possono } \\
\text { essere eseguite, dal personale non medico, con accuratezza anche durante il trasporto con l'ambulanza in movi- } \\
\text { mento. Viene evidenziata l'importanza di catturare un video di almeno } 6 \text { secondi per documentare le immagini } \\
\text { ecografiche, piuttosto che affidarsi a singole istantanee, al fine di migliorare la qualità dell'esame. } \\
\text { La percentuale (7,7\%) di immagini inadeguate è simile a quella di uno studio precedente dove le FAST pre-ospe- } \\
\text { daliere erano compiute da medici. Inoltre, il } 62 \% \text { delle FAST inadeguate aveva almeno una immagine adeguata } \\
\text { della tasca di Morison (area anatomica tra il fegato e il rene destro) o perisplenica o pelvica. } \\
\text { Lo studio riporta l'eccellente qualità del SonoSite MicroMaxx come ecografo portatile, grazie alla sua semplicità } \\
\text { d'uso e qualità di immagini. In definitiva i risultati della ricerca suggeriscono che l'ecografia pre-ospedaliera è una } \\
\text { metodica sicura ed affidabile anche se eseguita da personale non medico, poiché migliora i tempi di diagnosi e } \\
\text { trattamento di pazienti fortemente critici e, può apportare un importante contributo nell'identificazione del- } \\
\text { l'ospedale di destinazione di questi pazienti. }\end{array}$ \\
\hline Limiti & $\begin{array}{l}\text { - Lo studio non era randomizzato e prendeva in considerazione un campione di convenienza. } \\
\text { - A causa del numero ridotto di ecografi disponibili, vennero raccolti solo } 104 \text { ecografie. } \\
\text { - Bassa percentuale di professionisti arruolati nella ricerca e, nonostante ciò, molti di essi non completarono } \\
\text { - lo studio. } \\
\text { - ll campione di paramedici non era in cieco. Poiché sapevano di essere valutati, i lavoratori potrebbero aver } \\
\text { avuto uno stimolo in più a migliorare la propria performance. } \\
\text { La bassa percentuale di ecografie pre-ospedaliere positive può essere dovuto al fatto che il protocollo di } \\
\text { studio escludeva l'esecuzione di esami ecografici, qualora i soggetti riscontravano condizioni troppo insta- } \\
\text { bili per il paziente. } \\
\text { Potrebbe essere presente un bias di selezione causato dal reclutamento volontario del campione. Tale } \\
\text { aspetto comporta ad avere un pull di persone altamente motivati a migliorare la propria pratica professio- } \\
\text { nale. } \\
\text { Non esiste un corso specifico per addestrare tali professionisti all'esecuzione di ecografie d'urgenza. Per lo } \\
\text { studio sono state estrapolate le nozioni dalle linee guida dell'American College of Emergency Physician } \\
\text { (ACEP). } \\
\text { Non esiste conflitto di interesse per l'ecografo utilizzato nello studio: il SonoSite MicroMaxx, apparecchio } \\
\text { già utilizzato in altri studi precedenti che avevano l'obiettivo di valutare l'efficacia della FAST pre-ospeda- } \\
\text { liera eseguita da personale medico il quale è universalmente riconosciuto come il primo ecografo dedicato } \\
\text { al contesto pre-ospedaliero. Il SonoSite MicroMaxx ha le caratteristiche fondamentali per essere adeguato } \\
\text { a tale contesto: leggerezza, involucro resistente, sonda altamente versatile a più usi e produce immagini di } \\
\text { elevata qualità. }\end{array}$ \\
\hline
\end{tabular}

Tabella 2 - Secondo studio pertinente al quesito di ricerca (Heegaard et al., 2010) 


\begin{tabular}{|c|c|}
\hline Titolo & Emergency department ultrasound by nurse practitioners. \\
\hline Autori & Sean O. Henderson, Terence Ahern, David Williams, Thomas Mailhot, Diku Mandavia. \\
\hline $\begin{array}{c}\text { Rivista di pubblicazione } \\
\text { ed anno }\end{array}$ & Journal of the American Academy of Nurse Practitioners, 2010. \\
\hline Popolazione e contesto & $\begin{array}{l}\text { Il corso d'addestramento per eseguire ecografie d'urgenza rivolto ad infermieri del ED ed il follow-up dei pazien- } \\
\text { ti erano attuati presso il Centro Medico dell'Università del Sud California. L'ospedale di riferimento è un “Trauma } \\
\text { Center" di primo livello localizzato nella Downtown di Los Angeles. L'accesso annuale al ED è superiore a } 151.000 \\
\text { pazienti. }\end{array}$ \\
\hline Disegno di ricerca & $\begin{array}{l}\text { Lo studio è di tipo osservazionale ed è stato condotto prospettivamente in due step temporali. Il primo include- } \\
\text { va un corso di ecografia d'urgenza di due giorni ( } 16 \text { ore) e un anno di supervisione clinica. II secondo valutava } \\
\text { l'abilità degli infermieri del ED ad eseguire gli esami ecografici in un periodo di due mesi. }\end{array}$ \\
\hline Risultati & $\begin{array}{l}\text { Sono stati coinvolti } 5 \text { infermieri seguiti per un periodo di due mesi i quali hanno effettuato un totale di } 229 \text { esami } \\
\text { ecografici. } 2 \text { esami sono stati esclusi perché considerati non diagnostici in quanto le immagini erano di scarsa } \\
\text { qualità. In particolare sono state compiute: } 52 \text { ecografie renali, } 53 \text { della colecisti, } 51 \text { dell'aorta, } 42 \text { ecocardio- } \\
\text { grammi, } 25 \text { FAST e } 4 \text { ecografie ostetriche sottoposte alla revisione con un totale di } 27 \text { ecografie "positive". } \\
\text { 195( } 86 \%) \text { delle } 227 \text { immagini sono risultate adeguate. } 32 \text { immagini (14\%) sono considerate inadeguate: } 23 \text { trau- } \\
\text { mi addominali, } 3 \text { esami per aorta, } 3 \text { ecocardiogrammi, } 2 \text { esami per colecisti e } 1 \text { ecografia renale. La maggior parte } \\
\text { delle immagini inadeguate sono state "catturate" durante l'esame ad un paziente affetto da trauma addominale } \\
\text { in modo da verificare la presenza o l'assenza di liquido endo-addominale. Solo le immagini adeguate venivano } \\
\text { sottoposte ad approfondimento. I risultati mostrano che } 3 \text { ecografie riportano a falsi positivi e } 2 \text { ecografie con- } \\
\text { ducono a falsi negativi (individuavano o meno idronefrosi o calcolosi). Dalle analisi statistiche emerge che gli } \\
\text { infermieri hanno raggiunto un livello di sensibilità del } 93 \% \text { e di specificità del } 98 \% \text {. Con un training iniziale, gli } \\
\text { infermieri hanno identificato la presenza di patologia nel } 93 \% \text { dei casi ed esclusa nel } 98 \% \text {. Il valore predittivo posi- } \\
\text { tivo degli esami adeguati è stato calcolato pari all' } 89 \% \text { ed il valore predittivo negativo pari al } 99 \% \text {. I risultati di } \\
\text { questo studio provano l'efficacia di addestrare gli infermieri ad utilizzare l'ecografo. }\end{array}$ \\
\hline Discussione & $\begin{array}{l}\text { Secondo questo studio, in risposta all'aumento dei costi sanitari e dei tempi di attesa, i ED statunitensi hanno } \\
\text { assunto un maggiore numero di infermieri. Questi professionisti hanno progressivamente aumentato le loro } \\
\text { competenze specifiche avanzate al fine di soddisfare gran parte delle prestazioni non urgenti o semi-urgenti che } \\
\text { si presentano quotidianamente alla loro assistenza. In tale ottica, addestrare gli infermieri ad eseguire l'ecogra- } \\
\text { fia di urgenza è stato suggerito come uno dei metodi per ridurre i costi mantenendo la qualità assistenziale ero- } \\
\text { gata. } \\
\text { Questo è il primo studio che suggerisce l'insegnamento agli infermieri di eseguire ecografie d'urgenza al fine di } \\
\text { estendere questa metodica per rilevare patologie acute. }\end{array}$ \\
\hline Limiti & $\begin{array}{l}\text { - Gli infermieri partecipanti allo studio provenivano da un ospedale unico e non erano in cieco. La mancanza di } \\
\text { cecità può comportare alla presenza di bias di allocazione e di accertamento. } \\
\text { - Il campione ridotto ne limita la generabilità dei risultati. } \\
\text { - Tutte le immagini ecografiche erano riviste e giudicate da un singolo medico radiologo per tale motivo } \\
\text { potrebbero esserci degli errori metodologici. } \\
\text { - Il corso di addestramento è stato rivolto ad infermieri appartenenti ad un grosso ospedale universitario e } \\
\text { questo aspetto può influire nella motivazione, apprendimento degli infermieri studiati. }\end{array}$ \\
\hline
\end{tabular}

Tabella 3 - Terzo studio pertinente al quesito di ricerca (Henderson et al., 2010) 


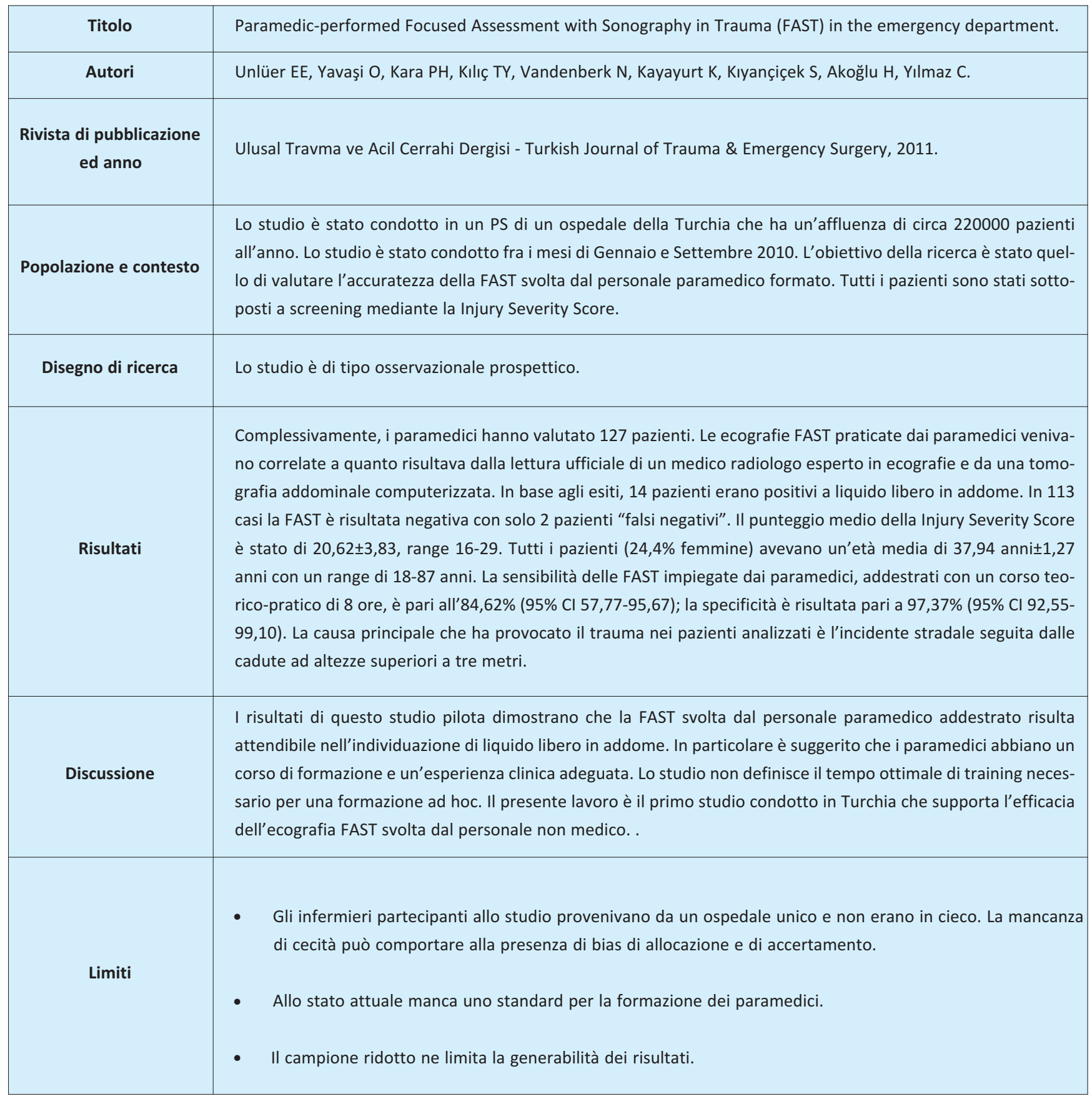

Tabella 4 - Quarto studio pertinente al quesito di ricerca (Unlüer et al., 2011)

\section{DISCUSSIONE}

Lo scopo principale della FAST è di identificare un'emorragia interna in un paziente politraumatizzato da avviare immediatamente al tavolo operatorio se emodinamicamente instabile oppure di considerare il paziente per approfondimenti diagnostici di secondo livello nel caso la stabilità dei parametri vitali lo consentano. I risultati di questa revisione sistematica rafforzano le recenti pubblicazioni che provano l'efficacia dell'ecografia FAST svolta dal personale sanitario, con assenza di specializzazione in radiodiagnostica, nella riduzione della mortalità nei pazienti affetti da trauma addominale chiuso. Tale efficacia ha dimostrato una sensibilità fra il 42-96\% ed una specificità fra l'85100\% (Rozycki et al., 1993; Cianci, 2009; Plummer et al., 1992; Buzio, et al., 2000; Ollerton et al., 2006; Rose, 2010; Patel \& Riherd, 2011). Analizzando l'intera letteratura scientifica nazionale ed internazionale sono quattro gli studi osservazionali, su 4767 documenti visualizzati, prettamente attinenti al quesito promosso nella presente revisione sistematica. Dai risultati riscontrati è possibile trarre alcune considerazioni che mettono in luce aspetti di clinica infermieristica ancora agli albori per poi essere discusse in futuro. Complessivamente sono state incluse 1035 ecografie 
FAST. In base agli studi considerati emerge che l'impiego dell'ecografia FAST eseguita dagli infermieri addestrati è molto efficace con una sensibilità che varia dall'84\% (95\% CI 72.1-92.2) (Bowra et al., 2010; Unlüer et al., 2011) al 93\% (Henderson et al., 2010) ed una specificità del 97,37\% (95\% CI 92.55-99.10) (Unlüer et al., 2011) al 98,4\% (95\% CI 94.9-99.6) (Bowra et al., 2010; Henderson et al., 2010). L'identificazione di una raccolta liquida in peritoneo può dipendere dall'operatore stesso, dalla tecnologia impiegata, dalla dinamica, dall'area di distribuzione del fluido e da possibili interferenze nella regione esplorata. In letteratura questo parametro varia da autore a casistica registrata, oscillando in un intervallo da 100 a $120 \mathrm{ml}$ fino a $500-600 \mathrm{ml}$, benché anche raccolte più piccole (20$50 \mathrm{ml}$ ) possono essere identificate se ben localizzate in aree facilmente accessibili agli ultrasuoni (Jehle et al., 2003; Cianci et al., 2008). L'accuratezza media della FAST, allo scopo di rilevare liquido libero, è stata simile per tutti quattro gli studi ed in particolare $86 \%$ (Henderson et al., 2010), 95\% (Bowra et al., 2010), 96\% (Unlüer et al., 2011) e 100\% (Heegaard et al., 2010). Riscontrando un'accuratezza elevata, i vantaggi dell'ecografia sarebbero comunque legati alla rapidità (tempi di esecuzione, disponibilità al letto del paziente, ecc.) e sicurezza (non invasività, riduzione di laparotomie inutili, minore esposizione a radiazioni, ecc.), con un impatto evidente sugli indicatori di salute del paziente critico (morbilità e mortalità) e gli indicatori di performance delle unità operative (tempi di diagnosi e trattamento, tempi di accesso alla sala operatoria, degenza e costi). Fra i quattro studi pertinenti è stato calcolato in un solo studio il tempo di esecuzione pratica della ecografia FAST con una media di 156 secondi (2,6 minuti), tempo mediano di 138 secondi (range $=76$ a 357). Le immagini inadeguate variano a seconda della pubblicazione ovvero per il 3,9\% (Unlüer et al., 2011) 4,0\% (Bowra et al., 2010), il 7,7\% (Heegaard et al., 2010) ed in fine il 14,0\% (Henderson et al., 2010). Negli studi considerati due trattavano specificatamente della figura infermieristica in PS e due analizzavano l'ecografia pre-ospedaliera eseguita da personale paramedico, non infermieristico. In buona parte del mondo occidentale, l'emergenza extra-ospedaliera è affidata alla figura del paramedico la quale è diversa da quella infermieristica sia per la tipologia di formazione scolastica che per l'impiego in ambito sanitario; mentre l'emergenza intra-ospedaliera, specie nel DE, è affidata all'infermiere con competenze specifiche di triage e trattamento del paziente critico. Nel nostro panorama l'emergenza intra ed extra-ospedaliera è affidata interamente alla figura infermieristica, in autonomia o in collaborazione con il medico. E' stato deciso comunque di prendere in considerazione lo studio di Heegaard (Heegaard et al., 2010) e la ricerca di Unlüer (Unlüer et al., 2011) poiché il paramedico può essere preso come termine di paragone per la gestione dell'emergenza preospedaliera eseguita da personale non medico, mediante protocolli valutativi di triage e procedure operative simili a quelli adoperati dall'infermiere operante nel sistema di emergenza territoriale, specie nel panorama europeo. Di notevole interesse è risultato che soltanto nello studio svolto dal personale paramedico le ecografie FAST venivano eseguite direttamente in ambulanza in movimento, quindi prima dell'accesso al PS. Infatti, allo stato attuale, non si è a conoscenza di esperienze che valutino le ecografie FAST svolte dagli infermieri durante il tragitto dall'ambulanza al PS o comunque nella pre-accettazione. Poiché in Italia, dopo l'abrogazione del mansionario, l'infermiere ha ottenuto maggiori e migliori riconoscimenti con competenze sempre più avanzate (Sansoni, 2007), gli autori di questa revisione sistematica hanno sentito l'esigenza di approfondire questa tematica credendo di fornire un iniziale e importante contributo alla letteratura dell'ecografia FAST infermieristica italiana. La ridotta disponibilità di studi ha reso possibile alcune riflessioni che potrebbero essere oggetto di ulteriori approfondimenti sintetizzati e qui sotto riportati:

- quale sarà l'impatto clinico che porterà l'ecografia FAST infermieristica in termini di miglioramento dell'assistenza;

- quale sarà la metodologia didattica più efficace e meno costosa per formare il personale infermieristico verso tale competenza avanzata;

- qual è il risparmio economico che l'azienda sanitaria avrà sposando questa metodica.

E' evidente che sarebbero necessari ulteriori studi, possibilmente RCT multicentrici che appurassero $\mathrm{i}$ risultati riscontrati fino ad ora. A tali studi andrebbe suggerito di includere un maggior numero di esami ecografici attraverso il coinvolgimento di infermieri di varia esperienza ed età deverse. Nonostante ciò, i primi risultati trovati in letteratura sono confortanti e possibile teatro di una futura ed eventuale sperimentazione in tale senso.

\section{LIMITI DELLA REVISIONE}

Ci sono diversi ostacoli nell'attuale revisione sistematica che potranno essere affrontati in future esperienze. Come in ogni revisione sistematica è possibile che non siano state identificate alcune riviste inerenti l'argomento trattato. Tuttavia, la ricerca ha considerato più database e gli autori hanno collaborato con un bibliotecario per affrontare la tematica nel modo più completo. Questa pubblicazione non ha incluso la letteratura inedita (ad es. atti di convegni, ecc.), ma è possibile che in questa possano essere presenti articoli rile- 
vanti al presente quesito di ricerca. L'esito della ricerca ha mostrato solamente quattro studi difatti un numero esiguo per raccomandare fermamente l'impiego della FAST infermieristica.

Infine, non è stato possibile associare i risultati fra tutti quattro i documenti rilevanti in quanto in alcuni di essi mancavano misurazioni statistiche come l'intervallo di confidenza; gli autori degli studi interessati sono stati contattati ma senza esito.

\section{CONCLUSIONE}

In conclusione, sta maturando la convinzione che l'ecografia point-of-care, ed in particolare la FAST svolta da infermieri, possono avere un ruolo molto importante nell'ambito delle emergenze e nei processi specifici di triage in quanto vero e proprio strumento di screening.

L'assegnazione del codice di gravità e priorità di trattamento, in stretta sinergia con le possibilità di cura, rappresenta un passaggio valido nel processo di triage, al fine di garantire l'accesso dei pazienti al percorso a loro più congruo. Questa revisione sistematica ha permesso di provare l'efficacia dell'ecografia FAST svolta dall'infermiere addestrato in termini di riduzione della mortalità e accuratezza della prestazione effettuata sul paziente affetto da trauma addominale chiuso.

\section{FONDI}

In tutte le fasi della revisione sistematica gli autori non hanno ricevuto contributi.

\section{CONFLITTO D'INTERESSE}

Gli autori dichiarano l'assenza di conflitti d'interesse.

\section{RUOLI E RESPONSABILITA'}

Per la realizzazione della revisione sistematica è stato necessario un lavoro di sinergia fra i tre autori. In particolare le loro funzioni sono state suddivise secondo il seguente modus operandi: MS è l'infermiere esperto di metodologia della ricerca ed è il responsabile della presente revisione sistematica. Le funzioni di MS sono state quelle di progettazione, pianificazione ed attuazione di tutte le fasi della ricerca; primo valutatore dei documenti reperiti; stesura della revisione sistematica e contatti con la redazione della rivista. LM è l'infermiere esperto dell'argomento. Le sue funzioni sono state: presentazione del quesito clinico e supporto nella ricerca dei documenti. LM è stato il secondo valutatore. VC è il medico esperto di ecografia d'urgenza e riconosciuto a livello internazionale per l'argomento trattato. VC è stato il supervisore in tutte le fasi e ha contribuito come terzo valutatore in alcuni documenti selezionati. Tutti gli autori hanno esperienza nell'argomento trattato e tutti approvano la versione finale della presente revisione sistematica.

\section{BIBLIOGRAFIA}

Bowra, J., Forrest-Horder, S., Caldwell, E., Cox, M., \& D'Amours, S.K. (2010). Validation of nurseperformed FAST ultrasound. Injury, 41 (5), 484-487.

Brouwers, M.C., Kho, M.E., Browman, G.P., Burgers, J.S., Cluzeau, F., Feder, G., Fervers, B., Graham, I.D., Hanna, S.E., Makarski, J. \& AGREE Next Steps Consortium. (2010). AGREE Next Steps Consortium. Development of the AGREE II, part 1: performance, usefulness and areas for improvement. CMAJ, 182 (10), 1045-1052.

Brouwers, M.C., Kho, M.E., Browman, G.P., Burgers, J.S., Cluzeau, F., Feder, G., Fervers, B., Graham, I.D., Hanna, S.E., Makarski, J. \& AGREE Next Steps Consortium. (2010). AGREE Next Steps Consortium. Development of the AGREE II, part 2: assessment of validity of items and tools to support application. CMAJ, 182 (10), 472-478.

Buzio, M., Bertello, A., Baù, G., \& Mao, P. (2000). Ultrasound in trauma (FAST). Minerva Chirurgica, 55 (1-2), 89-95.

Cartabellotta, A. (2011). AGREE II: appraising the clinical practice guidelines. Recenti Progressi in Medicina, 102 (5), 217-219.

Cianci, V. (2009). Handbook di ecografia in emergenzaurgenza. Quando il tempo conta. Manuale operativo-II edizione. Torino: Edizioni Medico-Scientifiche.

Cianci, V., Scuderi, M., Storti, E., \& Neri, L: (2008). FAST e il modello integrato di gestione ABCDE del trauma In Ecografia Clinica nelle Urgenze-Emergenze. Torino: Edizioni Minerva Medica.

Cole, F.L. (2003). Advanced practice nursing in the US: an overview. Emergency nurse, 11 (5), 22-25.

Heegaard, W., Hildebrandt, D., Spear, D., Chason, K., Nelson, B., \& Ho, J. (2010). Prehospital ultrasound by paramedics: results of field trial. Academy Emergency Medicine, 17 (6), 624-630.

Henderson, S.O., Ahern, T., Williams, D., Mailhot, T., \& Mandavia D. (2010). Emergency department ultrasound by nurse practitioners. Journal of the American Academy of Nurse Practitioners, 22 (7), 352355.

Kane, G.C., Hepinstall, M.J., Kidd, G.M., Kuehl, C.A., Murphy, A.T., Nelson, J.M., Schneider, L., Stussy, V.L., Warmsbecker, J.A., Miller, F.A. Jr, Pellikka, P.A., \& McCully, R.B. (2008). Safety of stress 
echocardiography supervised by registered nurses: results of a 2-year audit of 15,404 patients. Journal of the American Society of Echocardiography, 21 (4), 337341.

Lee, B.C., Ormsby, E.L., McGahan, J.P., Melendres, G.M., \& Richards, J.R. (2007). The utility of sonography for the triage of blunt abdominal trauma patients to exploratory laparotomy. American journal of roentgenology, 188 (2), 415-421.

Melniker, L.A., Leibner, E., McKenney, M.G., Lopez, P., Briggs, W.M. \& Mancuso, C.A. (2006). Randomized controlled clinical trial of point-of-care, limited ultrasonography for trauma in the emergency department: the first sonography outcomes assessment program trial. Annals of emergency medicine, 48 (3), 227-235.

Miles, G., Salcedo, A., \& Spear, D. (2011). Implementation of successful registered nurse peripheral ultrasound-guided intravenous catheter program in an Emergency Department. Journal of Emergency nursing.

Moher, D., Cook, D.J., Eastwood, S., Olkin, I., Rennie, D., \& Stroup, D.F. (1999). Improving the quality of reports of meta-analyses of randomised controlled trials: the QUOROM statement. Quality of Reporting of Meta-analyses. Lancet, 354 (9193), 1896-1900.

Moher, D., Schulz, K.F., \& Altman, D.G. (2001). The CONSORT statement: revised recommendations for improving the quality of reports of parallel-group randomised trials. Lancet, 357 (9263), 1191-1194.

Ollerton, J.E., Sugrue, M., Balogh, Z., D'Amours, S.K., Giles, A., \& Wyllie P. (2006). Prospective study to evaluate the influence of FAST on trauma patient management. Journal of trauma, 60 (4), 785-791.

Patel, N.Y., \& Riherd, J.M. (2011). Focused assessment with sonography for trauma: methods, accuracy, and indications. The Surgical clinics in North America, 91 (1), 195-207.
Plummer, D., Brunette, D., Asinger, R., \& Ruiz, E. (1992). Emergency department echocardiography improves outcome in penetrating cardiac injury. Annals of Emergency Medicine, 21 (6), 709-712.

Rose, J.S. (2010). Ultrasound in abdominal trauma. Emergency medicine clinics in North America, 22 (3), 581-599.

Rozycki, G.S., Ochsner, M., Jaffin, J., \& Champion, H.R. (1993). Prospective evaluation of surgeons' use of ultrasound in the evaluation of trauma patients. The journal of trauma, 34 (4), 516-527.

Ryan, J.J., Kyes, F.N., Horner, W.R., Young, J.C., \& Diamond, D.L. (1986). Critical analysis of open peritoneal lavage in blunt abdominal trauma. American journal of surgery, 151 (2), 221-223.

Sansoni, J. (2007). Nursing advanced practice: some thoughts. Professioni Infermieristiche, 60 (1), 5-12.

Storti, M., Cappozzo, S., Pegoraro, F., Pozza, B., \& Rossetto, V. (2011). Efficacia della supervisione clinica. Leadership Medica, 283 (1), 16-23.

Unlüer, E.E., Yavaşi, O., Kara, P.H., Kılıç, T.Y., Vandenberk, N., Kayayurt, K., Kıyançiçek, S., Akoğlu, H., \& Yilmaz, C. (2011). Paramedicperformed Focused Assessment with Sonography in Trauma (FAST) in the emergency department. Ulusal Travma ve Acil Cerrahi Dergisi, 17 (2), 113-116.

von Elm, E., Altman, D.G., Egger, M., Pocock, S.J., Gøtzsche, P.C., \& Vandenbroucke, J.P. (2007). STROBE Initiative. Strengthening the Reporting of Observational Studies in Epidemiology (STROBE) statement: guidelines for reporting observational studies. British Medical Journal, 335 (7624), 806808.

Walcher, F., Weinlich M., Conrad, G., Schweigkofler, U., Breitkreutz, R., Kirschning, T., \& Marzi, I. (2006). Prehospital ultrasound imaging improves management of abdominal trauma. The British journal of surgery, 93 (2), 238-242. 